Home Browse Inderscience Publishers Orders Authors Librarians

Enter words / phrases / DOI / ISSN / authors / keywords / etc.

Home > International Journal of Intelligent Enterprise $>$ List of Issues > Volume 7, Issue 4

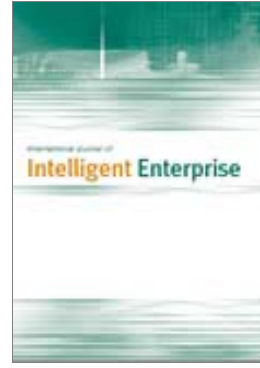

$<$ Previous issue

\section{International Journal of Intelligent Enterprise}

Print ISSN: $1745-3232$ Online ISSN: $1745-3240$

- Current issue

- Subscribe

- Get TOC alerts

- About this journal
- List of issues

\section{Volume 7, Issue 4}

Select All For selected items: Please select

Incorporating carbon emissions in queuing models to determine lot sizes and inventory buffers in a supply chain

Petrus Setya Murdapa, I. Nyoman Pujawan, Putu Dana Karningsih, Arman Hakim Nasution

7(4), pp. 373-390

Keywords: carbon emission, inventory buffering, lot sizing, queuing, performance model, supply chain

Abstract | PDF (469 KB)

Entrepreneurial passion facing its ecosystem's obstacles: the case of

\section{Tunisia}

Samira Boussema

7(4), pp. 391-404

Keywords: entrepreneurial passion, unimplementation of projects, structural modelling

Abstract | PDF (248 KB)

Implementing just-in-time-based supply chain for the bulk items in an integrated steel plant

Ram Naresh Roy

7(4), pp. 405-422

Keywords: JIT supply chain, logistics, bulk materials, integrated steel plant, MRP model, costsavings and productivity, lean procurement

Abstract | PDF (310 KB)

Framework to identify a set of univariate time series forecasting techniques to aid in business decision making

Iram Naim, Tripti Mahara

7(4), pp. 423-443

Keywords: univariate time series, time series pattern, model selection, trend analysis, seasonal data, complex seasonality, long time series

Abstract | PDF (836 KB)

Innovativeness, environment and performance of small and medium-sized enterprises in manufacturing sector in Malaysia

Mandy Mok Kim Man, Lo May Chiun

7(4), pp. 444-460

Keywords: innovativeness, small and medium-sized enterprises, SMEs, environment, performance, technology, entrepreneurship
Most Read Most Cited

Real time noisy dataset implementation of optical character identification using CNN

Comparative study on IDS using machine learning approaches for software defined networks

Indexing documents with reliable indexing techniques using Apache Lucene in Hadoop

The effect of lean on job satisfaction

Influence of human resource management practices on the organisational commitment with specific reference to selected hotels in Chennai

See More 
Abstract | PDF (299 KB)

Modelling for system fluctuations advancing buffer management

delivering on the theory of constraints

J. Reddy, A. Telukdarie

7(4), pp. 461-480

Keywords: manufacturing systems, Industry 40 , theory of constraints, TOC, multimethod

simulation modelling, production optimisation

Abstract | PDF (421 KB)

Impact of social media advertising on millennials buying behaviour

Taanika Arora, Arvind Kumar, Bhawna Agarwal

7(4), pp. 481-500

Keywords: social media, millennials, informativeness, entertainment, credibility, interactivity, privacy concerns, social media advertising, attitude, behavioural responses, buying behaviour, Indians

Abstract | PDF (287 KB)

\section{Keep in touch:}

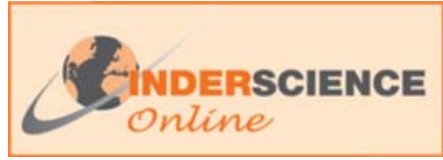

Inderscience Online

Advanced Search

Browse

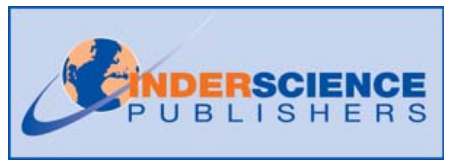

Inderscience Publishers

Subscribe

Authors

Librarians

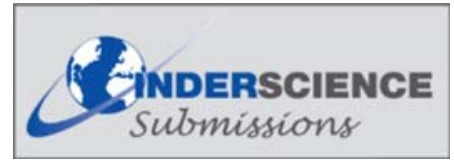

Inderscience Submissions

Submissions Guidelines

Submit an Article 


\title{
Incorporating carbon emissions in queuing models to determine lot sizes and inventory buffers in a supply chain
}

\section{Petrus Setya Murdapa}

Department of Industrial Engineering, Institut Teknologi Sepuluh Nopember (ITS),

Surabaya, Indonesia

and

Department of Industrial Engineering,

Widya Mandala Catholic University,

Madiun, Indonesia

Email: setya.murdapa@gmail.com

\section{Nyoman Pujawan* and Putu Dana Karningsih}

Department of Industrial Engineering, Institut Teknologi Sepuluh Nopember (ITS),

Surabaya, Indonesia

Email: pujawan@gmail.com

Email: putu.karningsih@gmail.com

*Corresponding author

\section{Arman Hakim Nasution}

Department of Business Management, Institut Teknologi Sepuluh Nopember (ITS),

Surabaya, Indonesia

Email: armanhakim.nasution@gmail.com

\begin{abstract}
In this paper, we present a supply chain model that considers both inventory-related costs and emissions. We used the queueing-based performance model wherein emissions in three stages of supply chain activities are captured. The model was solved by the decomposition approach. For model validation, we have used a discrete event simulation. The computation results show that the two results, i.e., the decomposition approach and the simulation, are very close, indicating the accuracy the approach that we used. Experiments were conducted to test the applicability of the model. The numerical examples show that the change in parameter values is not always responded the same way by the total inventory-related costs and the emission costs, indicating the importance of including these two response variables in the model.
\end{abstract}

Keywords: carbon emission; inventory buffering; lot sizing; queuing; performance model; supply chain. 
Reference to this paper should be made as follows: Murdapa, P.S., Pujawan, I.N., Karningsih, P.D. and Nasution, A.H. (2020) 'Incorporating carbon emissions in queuing models to determine lot sizes and inventory buffers in a supply chain', Int. J. Intelligent Enterprise, Vol. 7, No. 4, pp.373-390.

Biographical notes: Petrus Setya Murdapa is currently a $\mathrm{PhD}$ candidate in Logistics and Supply Chain Engineering in the Department of Industrial Engineering at Institut Teknologi Sepuluh Nopember (ITS), Surabaya, Indonesia. He received his undergraduate degree in Department of Chemical Engineering from Gadjah Mada University Yogyakarta and Master in Industrial and Systems Engineering from AIT Bangkok, Thailand. He is a Lecturer in the Department of Industrial Engineering, Widya Mandala Catholic University, Madiun, Indonesia.

I Nyoman Pujawan is a Professor of Supply Chain Engineering in Institut Teknologi Sepuluh Nopember (ITS), Surabaya, Indonesia. He received his Bachelor in Industrial Engineering from ITS, Indonesia, Master of Engineering in Industrial Engineering from Asian Institute of Technology (AIT) Bangkok, Thailand, and PhD in Management Science from Lancaster University, UK. He was a Lecturer in Operations Management in Manchester Business School, The University of Manchester, UK in 2003-2004. His papers have appeared in many international journals including the European Journal of Operational Research, International Journal of Production Economics, International Journal of Production Research, among others.

Putu Dana Karningsih is an Assistant Professor of Manufacturing System at the Department of Industrial Engineering, Institut Teknologi Sepuluh Nopember (ITS), Surabaya, Indonesia. She is currently the Head of Manufacturing System Laboratory in ITS. She received her Bachelor in Industrial Engineering from University of Indonesia, Master and $\mathrm{PhD}$ in Mechanical and Manufacturing Engineering from the University of New South Wales (UNSW), Australia. Her research interests are in lean production, manufacturing systems, supply chain risk management and collaborative product and process development.

Arman Hakim Nasution is an Associate Professor of Business and Strategic Management at the Department of Business Management, Institut Teknologi Sepuluh Nopember (ITS), Surabaya, Indonesia. He received his Bachelor in Industrial Engineering from ITS, Master of Engineering in Industrial and Systems Engineering from AIT Bangkok, Thailand, and $\mathrm{PhD}$ in Industrial Engineering from Gadjah Mada University, Jogjakarta, Indonesia. His research interest is in policy alignment analysis.

\section{Introduction}

Currently, the world is facing the problem of greenhouse gasses (GHG) whose concentration in the atmosphere continues to rise due to the emission process from various sources. Because GHG is acidic and retains heat in the atmosphere (Carbonfootprint.com, n.d.; Wang and Choi, 2015b), the high concentration of GHG leads to acid rain and global warming. The subsequent consequences are climate change, sea water flood due to polar ice melting, changes or even ecosystem extinction in the sea due to coral reef damage, as well as on land and air (US Environmental Protection 
Agency, 2016). These damaging consequences have invited attention of world leaders to take actions in order to reduce the high concentrations of GHG or its rate of increase in the atmosphere. It began with the establishment of the Intergovernmental Panel on Climate Change (IPCC) in 1988 and continued with the convening of world leaders meetings. Some of the most important were the Earth Summit in Rio de Janeiro in 1992, that in Kyoto, Japan, in 1997, which produced the Kyoto Protocol, and in Paris, which produced the Paris Agreement in 2015.

The Kyoto Protocol proposed three emission reduction mechanisms, namely the international emission trading scheme (which includes carbon tax, emission cap, cap and trade, etc.), joint implementation (JI), and clean development mechanism (CDM) (Ratnatunga et al., 2012). The Paris Agreement, which has been signed by some 195 countries and ratified by some 170 countries, set ambitious targets, among others, to seek global temperatures not to exceed $2^{\circ} \mathrm{C}$ above the pre-industrial (1750) temperature by reducing the amount of emissions. These countries declared their determination to take responsibility and participate in reducing carbon emissions to zero by 2100 .

The participation of countries to ratify both the Kyoto Protocol and the Paris Agreement meant that the industries in those countries must limit their emissions. Emission-induced industrial activities should be regulated so that the industry can support the objective of reducing the emissions while remaining competitive in the market. Industrial supply chain activities, such as production, shipment of materials and finished goods, as well as the storage and material handling, are all associated with emissions. Emission restrictions, by applying emission cap mechanisms, carbon taxes, or cap and trade, will affect strategic decisions such as which factories or warehouses need to be opened or closed (Fahimnia et al., 2015a) as well as operational decisions such as order quantity or production lot (Fahimnia et al., 2015b; Wang and Choi, 2015b). An important implication is that all decisions pertinent to the supply chain should take into account the emission factors in addition to the financial aspects. Decision, design, or control variables should be chosen in such a way as without violating emissions quota constraints and taking into account emissions tax or taking advantage of cap and trade mechanisms.

Academics have made many attempts to include emissions as one of the factors when making supply chain decisions. However, there is still a lot of work to be done in this respect. According to Altiok (1997), there are, in general, two types of models related to production or supply chain systems, the first is the optimisation models and the second is the performance models. The first one is mostly based on mathematical programming while the second is mainly based on the queuing theory. While there are many optimisation-type models that take into account emissions, virtually no performance-type model does so. The optimisation model is intended for the selection of the best control settings that involve emissions as an objective function or as a constraint. Most are mathematical programming, like those published by Benjaafar et al. (2012), Pan et al. (2013), Shaw et al. (2013), Fahimnia et al. (2015b), Brandenburg (2015) and Zakeri et al. (2015). The other stream that has also taken emission into account is the economic order quantity (EOQ) model or its variations. Among others, papers in this category are Hua et al. (2011), Chen et al. (2013), Arslan and Turkay (2013), Toptal et al. (2014) and Toptal and Çetinkaya (2015).

Both types of models, the performance model and the optimisation model, have advantages and disadvantages, but both are in different usage time windows. Logically, the performance model will be used first, and then the optimisation model can be 
developed using the structured performance model. This is seen in Karaman and Altiok (2009).

Almost all papers on performance models, in both the production line and supply chain schemes, have not paid attention to the emission aspect. To the best of our knowledge, only papers written by Wang and Choi (2015a, 2015b) incorporate emissions in their performance, queuing-based models. The models of Wang and Choi (2015a, 2015b) address the lot-sizing decisions of batch manufacturing systems in a make-to-order mechanism under stochastic demand conditions. These two papers discussed two systems of carbon emissions trading, namely cap and trade and tax policy. Recently, Murdapa et al. (2018) tried to add emission quantities to the classic model of single stage queue/manufacturing. Although still in a very simple way, the paper utilised an existing performance model for system analysis that takes into account emissions, under a carbon tax mechanism.

A performance-based model that takes into account emissions should be developed; at the least it must able to emphasise the concept of how to add emission quantities to a performance model. There have been many performance models for analysing various types of production line systems, but there are still few that add the amount of emissions into the model. Given that a performance model is able to describe how a system behaves/processes, it is logical to observe how emissions emerge through that performance model. Given that the supply chain is essentially a gradual queuing system, this paper will use the performance model as the basis for inventory analysis along the supply chain. The objective is to obtain stock and replenishment decisions in a simple supply chain system that consists of a retailer ordering items from a manufacturer in order to satisfy demand from customers and the manufacturer orders materials from the supplier. As in previous papers on multi-stage production line and multi-echelon supply chain (Dallery and Gershwin, 1992; Karaman and Altiok, 2009; Saetta et al., 2012), we employ a method of decomposition in the analysis. The original system is broken up into several two machines-one buffer (2M1B) subsystems. Then, the subsystems are coupled back with a linking equation called the decomposition equation. This paper will accommodate the Dallery and Frein (1993) technique combined with the system structure at Karaman and Altiok (2009). Our major question would be to address the reordering and stocking decisions in the supply chain where emissions are taken into account.

This paper will deliberately simplify the performance model for the supply chain system developed by Karaman and Altiok (2009), which originally assumed that the duration of time in the system followed a phase-type distribution, becoming a simple exponential distribution. The decomposition techniques that were originally used in the context of production line systems (Dallery and Frein, 1993) will be extended into the case of the supply chain system of the study of Karaman and Altiok (2009). However, the main objective is to include the quantity of emissions that emerge, into the model when the system operates. The quantity of emissions can be accommodated into the model in the form of a response variable. The novelty of this paper is more about using performance models for supply chain systems with the decomposition techniques of Dallery and Frein (1993), while taking into account the amount of emissions.

In the following sections, we will describe the systems, the development and validation of the model, and numerical examples. Validation will be done by the use of simulation. The discussion of the results and the conclusions will then be presented. 


\section{System description}

We address a simple supply chain system consisting of a supply company, a factory, and a retailer, as shown in Figure 1. We modified the system in Karaman and Altiok (2009) where here we omit the echelon distribution centre. The system operates under certain lot-sizes and inventory buffer policies, which are represented by the combination of control variables $\left(R_{R M}, Q_{R M}\right),(R, r),\left(R_{R}, Q_{R}\right)$. Customer demand is fulfilled by retailers from stock available in $R T$ stock. Fulfilment of demand causes the stock to decrease gradually. Stock is controlled by $\left(R_{R}, Q_{R}\right)$ mechanism where $R_{R}$ and $Q_{R}$ are reorder point and order quantity, respectively. The order is submitted to the factory, which maintains finished goods inventory in $F G$ stock. The time required for the delivery of products from the factory to the retailer is $T T_{R T}$.

Figure 1 Supply chain system prototype discussed in this paper (see online version for colours)

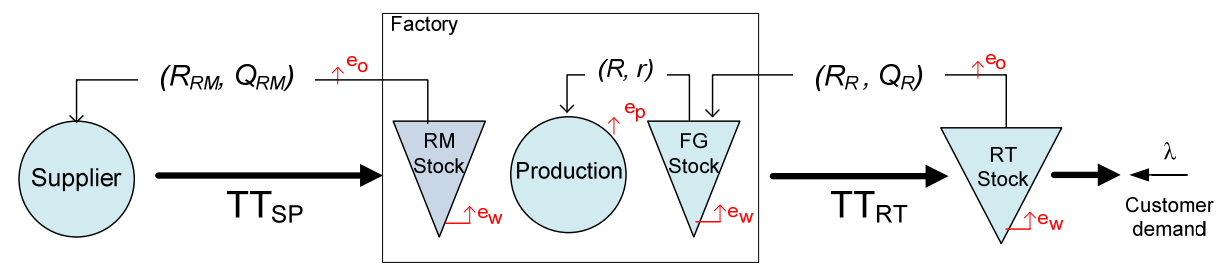

In the meantime, the manufacturer uses a $(R, r)$ mechanism in controlling the inventory of finished product, where $r$ is the reproduction point and $R$ is the targeted inventory in FG stock. It is assumed that there will be no set-up cost at each production start when inventory is equal or less than $r$. The required raw materials are ordered to the supplier. The stock of raw materials at RM stock is controlled using the $\left(R_{R M}, Q_{R M}\right)$ mechanism. Every shipment of raw materials from supplier to factory takes $T T_{S P}$ time unit.

As long as the supply chain system in Figure 1 operates, emissions appear to accompany the following processes. Procurement of raw materials begins with the submission of orders by the factory followed by the delivery of goods from the supplier. Similarly, the ordering of finished products by retailers to the factory. It is assumed that every order is always followed by one shipment. Therefore, every order will bring emissions due to transportation. The amount of emission is $\varepsilon_{O} \mathrm{~kg}$ carbon/order. Next, the storage of goods, both raw materials at RM stock and finished products in FG stock and RT stock, is assumed to use electrical energy to maintain the condition and quality of goods. Thus, there is an emission expressed in $\mathrm{kg}$ carbon for each unit of goods stored for each period, $\varepsilon_{H} \mathrm{~kg}$ carbon/unit/time unit. Another activity that is very obvious to cause emissions is production. Emissions occur when production activities use electrical energy to drive the production machine. Or, it could be because the production process itself produces emissions. Production emissions are expressed in $\varepsilon_{P} \mathrm{~kg}$ carbon/unit. Each kilogram of carbon causes the system to pay a tax (carbon tax) of $C_{\varepsilon} \mathrm{Rp} / \mathrm{kg}$ carbon. The values for each control variable: $R_{R M}, Q_{R M}, R, r, R_{R}$ and $Q_{R}$ must be decided, so that the cost of inventory and emissions can be as low as possible. Selection of those values will also determine the minimum required capacity of RM stock, FG stock, and RT stock. 


\section{Model development}

\subsection{Dallery-Frein model adoption}

In the queuing system, we need to define the probability distribution of inter-arrival time of the jobs or customers as well as the service time. Most of the queuing models use the assumption of exponential distribution for both the inter-arrival time as well as the service time. As an example, Dallery and Frein (1993) used the assumption of exponential distribution for flowline system analysis. Certainly, other probability distribution has also been used. In the discrete version, Gershwin (1987) and his subsequent papers and colleagues used geometric distribution for the design of transfer line systems.

Table 1 Validation

\begin{tabular}{|c|c|c|c|c|c|c|c|}
\hline$R_{R M}=10$ & \multicolumn{2}{|c|}{$Q_{R M}=15$} & $r=10$ & $R=30$ & $R_{R}=$ & & $Q_{R}=10$ \\
\hline$T T_{S P}=50$ & & & \multicolumn{2}{|c|}{ rate $=0.125$} & \multicolumn{3}{|c|}{$T T_{R T}=50$} \\
\hline \multicolumn{4}{|c|}{ (a) Before adjustment } & \multicolumn{4}{|c|}{ (b) After adjustment } \\
\hline & \multicolumn{3}{|c|}{ Average stock at } & & \multicolumn{3}{|c|}{ Average stock at } \\
\hline & $\begin{array}{c}R M \\
\text { stock }\end{array}$ & $\begin{array}{c}F G \\
\text { stock }\end{array}$ & $\begin{array}{c}R T \\
\text { stock }\end{array}$ & & $\begin{array}{c}R M \\
\text { stock }\end{array}$ & $\begin{array}{c}F G \\
\text { stock }\end{array}$ & $\begin{array}{c}R T \\
\text { stock }\end{array}$ \\
\hline \multicolumn{8}{|c|}{$\lambda=0.03$} \\
\hline Model & 17.9 & 19.92 & 10.33 & Model & 16.96 & 22.48 & 9.46 \\
\hline Sim & 16.5 & 22.57 & 9.02 & Sim & 16.5 & 22.57 & 9.02 \\
\hline Rel. error & $7.82 \%$ & $-13.31 \%$ & $12.68 \%$ & Rel. error & $2.71 \%$ & $-0.40 \%$ & $4.65 \%$ \\
\hline \multicolumn{8}{|c|}{$\lambda=0.025$} \\
\hline Model & 17.92 & 20.31 & 10.36 & Model & 16.98 & 22.92 & 9.49 \\
\hline Sim & 16.75 & 22.95 & 9.27 & Sim & 16.75 & 22.95 & 9.27 \\
\hline Rel. error & $6.53 \%$ & $-13.00 \%$ & $10.52 \%$ & Rel. error & $1.35 \%$ & $-0.13 \%$ & $2.32 \%$ \\
\hline \multicolumn{8}{|c|}{$\lambda=0.02$} \\
\hline Model & 17.93 & 20.68 & 10.39 & Model & 16.99 & 23.34 & 9.51 \\
\hline Sim & 16.96 & 23.28 & 9.53 & Sim & 16.96 & 23.28 & 9.53 \\
\hline Rel. error & $5.41 \%$ & $-12.56 \%$ & $8.28 \%$ & Rel. error & $0.18 \%$ & $0.26 \%$ & $-0.21 \%$ \\
\hline \multicolumn{8}{|c|}{$\lambda=0.015$} \\
\hline Model & 17.95 & 21.04 & 10.42 & Model & 17 & 23.74 & 9.54 \\
\hline Sim & 17.29 & 23.72 & 9.78 & Sim & 17.29 & 23.72 & 9.78 \\
\hline Rel. error & $3.68 \%$ & $-12.75 \%$ & $6.14 \%$ & Rel. error & $-1.71 \%$ & $0.08 \%$ & $-2.52 \%$ \\
\hline \multicolumn{8}{|c|}{$\lambda=0.01$} \\
\hline Model & 17.97 & 21.37 & 10.45 & Model & 17.02 & 24.12 & 9.57 \\
\hline Sim & 17.45 & 24.09 & 9.97 & Sim & 17.45 & 24.09 & 9.97 \\
\hline Rel. error & $2.89 \%$ & $-12.71 \%$ & $4.59 \%$ & Rel. error & $-2.53 \%$ & $0.12 \%$ & $-4.18 \%$ \\
\hline
\end{tabular}


This paper adopts the Dallery and Frein (1993) technique, so that production time, transport time, and time between customer arrivals are assumed to be exponentially distributed. Table 1 lists a brief overview of the basic differences in this paper with Dallery and Frein (1993), Karaman and Altiok (2009) and Saetta et al. (2012). Since the Dallery and Frein (1993) model examines a serial flowline consisting of a machine-buffer-machine (2M1B) repeatedly, the supply chain system in this paper requires a small modification in order to be analogous to the Dallery-Frein system. First, the echelon supplier is considered to be a 'machine' with an average cycle time of $T T_{S P} / Q_{R M}$ (equivalently, the average rate is $Q_{R M} / T T_{S P}$ ). Second, a 'machine' representing the echelon retailer needs to be added, where $T T_{R T} / Q_{R}$ will be the cycle time (equivalently, $Q_{R} / T T_{R T}$ is the retailer machine average rate). The result of the modification is shown in Figure 2.

Figure 2 The modified prototype system (see online version for colours)

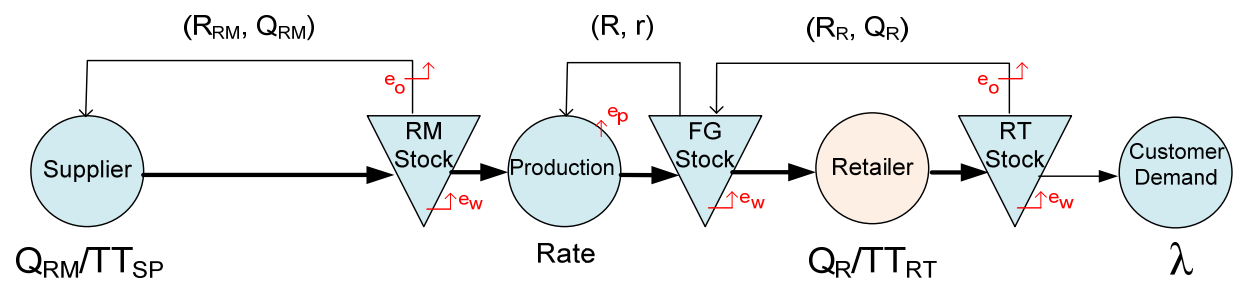

Figure 3 Decomposition of system S to subsystems $\mathrm{S}(1), \mathrm{S}(2)$ and $\mathrm{S}(3)$ (see online version for colours)

$\mathrm{S}$

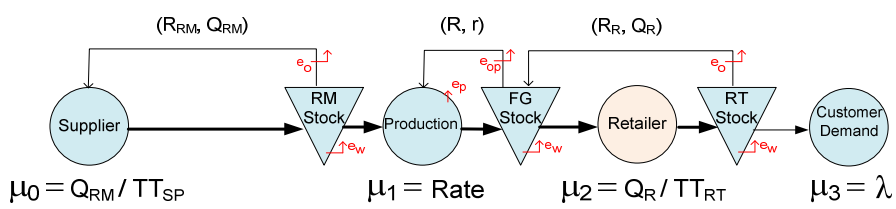

$\mathrm{S}(1)$

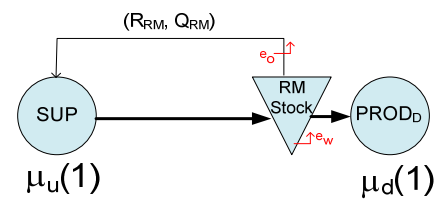

S(2)

S(3)
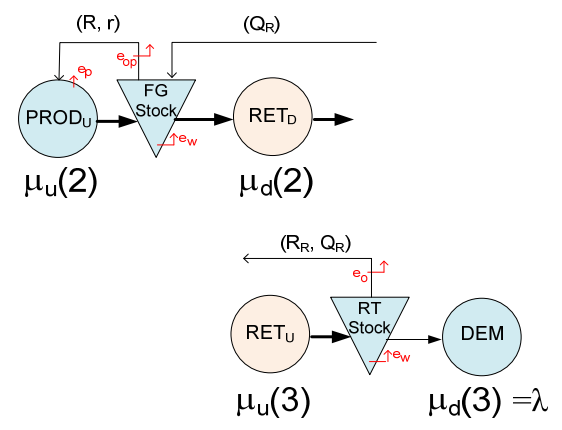
Figure 4 Dallery and Frein's algorithm (modified-A1b)

(Initialisation):

Set the first upstream machine average rate $\mu_{u}(1)=\mu_{0}$ and the last downstream average rate $\mu_{d}(1)=\mu_{1}, \mu_{d}(2)=\mu_{2}, \mu_{d}(3)=\mu_{3}$

Set the last subsystem throughput $X(3)$ and the next upstream machine average rate $\mu_{u}(i)$ for $i=2,3$ to some initial values

(Iteration):

STEP 1 For $i=1,2$

1.1 Calculate the instant probability $p(0, i), p(1, i)$ and blocking probability $p_{s}(i)$

$$
p_{s}(i)=p_{d}(0, i)=\frac{p(1, i)}{1-p(0, i)}
$$

1.2 Calculate the downstream machine average rate $\mu_{u}(i)$ using:

$$
\frac{1}{\mu_{u}(i+1)}=t_{u}(i+3)=t_{i}+p_{s}(i) t_{u}(i) \text { for } i=1,2
$$

STEP 2 Calculate the last subsystem throughput, $X(3)$, using:

$$
X(3)=\mu_{d}(3)(1-p(0,3))
$$

\section{STEP 3 For $i=2,1$}

3.1 Set the subsystem throughput $X(i)=X(3)$

3.2 Calculate the upstream machine average rate $\mu_{d}(i)$ using:

$$
\mu_{d}(i)=\frac{X(i)}{(1-p(0, i))}
$$

and the newest value of the instant probability $p(0, i)$ obtained from STEP 1.1

STEP 4 Return to STEP 1, until the values of unknown parameters have been converged.

The system in Figure 2 is then decomposed into three subsystems, as in Figure 3, with Figure 4 as the algorithm which will be used to aggregate them back to the original system. The original system is called $\mathrm{S}$, and the $\mathrm{i}^{\text {th }}$ subsystem is called $\mathrm{S}(\mathrm{i})$. Since $\mathrm{S}$ system has three buffers (RM stock, FG stock and RT stock), there will be three subsystems, i.e., S(1), S(2) and S(3). Each subsystem consists of two machines and one interstage buffer (2M1B). One machine is an 'upstream' machine, which has average rate of $\mu_{u}(i)$, and the other is a 'downstream' machine, which has average rate of $\mu_{d}(i)$. The buffer in the subsystem S(i) should exactly have the same characteristic as the corresponding buffer in the system $\mathrm{S}$.

Following the decomposition method proposed by Gershwin (1987), Dallery and Gershwin (1992) or Dallery and Frein (1993), the upstream machine (or downstream machine) of each subsystem is actually an aggregate of the upstream (or downstream) part of the system $\mathrm{S}$. The upstream machine $\mathrm{PROD}_{\mathrm{U}}$ in subsystem $\mathrm{S}(2)$, for example, is an aggregate of the production machine together with RM stock buffer and supplier machine. Meanwhile, the downstream machine $\mathrm{PROD}_{\mathrm{D}}$ in subsystem $\mathrm{S}(1)$ is an aggregate of production machine, FG stock buffer, retailer machine, RT stock buffer, and customer demand machine.

The analysis is carried out by assuming that the system has reached the steady state. Therefore, a state transition diagram of the subsystem S(i) can be constructed first so that 
a set of transition equations can then be generated, and completed to calculate the steady state probability of each state in the subsystem S(i). In the model, it is assumed that there may be backorder on RM stock, FG stock and RT stock buffer; hence, the state transition diagram for each subsystem can be constructed as seen in Figure 5, Figure 6, and Figure 7 respectively. In all three figures, each state-transition diagram is truncated to a certain level of negative state to avoid infinity. For a deeper explanation of decomposition, the reader is referred to the paper of Dallery and Frein (1993).

It turns out that the subsystems $\mathrm{S}(1)$ and $\mathrm{S}(3)$ are identical, in contrast to the $\mathrm{S}(2)$ subsystem. The steady state solution for the subsystem $S(1)$ and $S(3)$ are quite simple. Meanwhile, the steady state solution for subsystem $\mathrm{S}(2)$ is done by an approach, i.e. some states are combined into one so that it is imagined to operate in batches, as shown in Figure 6(c). In this paper all solutions are searched numerically.

The system $\mathrm{S}$ performance formulation is obtained by reconnecting the subsystems S(i) to each other. The method given by Dallery and Frein (1993) is quite accurate though simple. Given that supply chain activity will only take place if there is demand to the retailer, then the demand rate, $\lambda$, will determine the system throughput. Thus, among several algorithms provided by Dallery and Frein (1993), the Alb-algorithm is chosen for the computation process. Only a small modification is needed, i.e., reversing the sequence of throughput calculation. Originally from upstream to downstream (push), it is now converted from downstream to upstream (pull). See Figure 4 . Let $X(i)$ be the throughput of the subsystem $i$, in unit of entity per time unit.

\section{Validation}

Some validation is needed to ensure that the performance model, which is rearranged assuming an exponential distribution developed in this paper, is in line with the model in the reference. Thus, the calculation of the response value can be quite reliable. It is not easy to compare the performance of the exponential model to the phase-type model of Karaman and Altiok (2009) because their numbers of parameters are different. Hence, the modified-A1b algorithm is validated using the available ARENA simulation model adopted from Altiok (1997). This Altiok simulation model was originally a simulation model for a supply chain system with four echelons, i.e., supplier - production distribution centre (DC) - retailer. For validation purposes, the simulation model is simplified and adapted for an exponential three-echelon system.

Some steady state simulation runs give very small deviation results, hence they are said to be convergent. The comparison of the average inventory calculations on each buffer, based on the numerical model and simulation, is, thus, sufficiently expressed in its relative error value (as in Karaman and Altiok, 2009).

The comparison of the simulation results and those from the algorithm is presented in Table 1 . The values of average stock at RM stock and at RT stock show not so good proximity between those obtained from the proposed model and from the simulation. Also, much more apparent deviations were exhibited by the average stock in FG stock. This is similar to the results obtained by Karaman and Altiok (2009). The state transition diagram in FG stock buffer is much more complex than the diagram formulation in other buffers. Through the simulation results, it can be recognised that the related variances are very small, indicating that the incompatibility of the outcomes is systemic. Thus, we 
could propose a coefficient of multiplication in the algorithm. A multiplication of $94.73 \%$ for the RM stock, of $112.87 \%$ for the FG stock, and of $91.56 \%$ for the RT stock provided a very close fit to the simulation results. In most cases presented in Table 1, the relative error values are lower than 5\%. Overall, we would say that, after correction, the algorithm could work well.

Based on briefly calculated case examples, it can be reported that the use of the model is only limited to the value of the average demand that is not more than $+/-50 \%$ of the shipping rate, whether shipping from suppliers to factories, or shipments from factories to retailers. Outside of that range, the calculation results show a higher error.

\section{Incorporating emissions}

In a dynamic system, there are four types of variables, namely, state, control, nature, and response. In this study, the emission quantity will be captured in the form of a response variable. As is common in inventory models, the response variable could be in the form of the average inventory level, the inventory value, or the throughput of the system. Here we combined both inventory-related cost and emission cost as response variables. Inventory-related costs are those incurred in the shipment of materials and finished goods, production, and storage. In this section, we will model the total costs, which consist of inventory-related costs and emission costs for each stage of supply chain activity, i.e., the upstream side that concerns the material supply from supplier to factory, the internal stage which is production activity, and the downstream side that concerns the shipment of finish goods to the retailer.

\subsection{Supply of raw materials from supplier to factory}

State transition diagrams for supplying raw materials from suppliers to factory warehouses can be seen in Figure 5. Each time there is a state transition, emissions will occur. For example, a transition from state $R_{R M}-1$ to state $R_{R M}+Q_{R M}-1$ will generate order and hence transport emission of $\varepsilon_{O} \mathrm{~kg}$ carbon/order. Meanwhile, when the system is in a certain state, there will be storage emission $\varepsilon_{H} \mathrm{~kg}$ carbon/unit of product/time. When a transition occurs from state $R_{R M}+Q_{R M}$ to state $R_{R M}+Q_{R M}-1$ there is a production emission $\varepsilon_{P} \mathrm{~kg}$ carbon/product unit.

Total emissions, $E_{R M}$, can be formulated as follows:

$$
\begin{aligned}
E_{R M}= & \text { emissions due to raw material transport from supplier } \\
& + \text { emissions due to material storage at RM Stock }
\end{aligned}
$$

With reference to Taha (2007), it can be formulated that the ordering frequency is $\mu_{d}(1) / Q_{R M}$. Whereas, the average inventory can be calculated based on the steady state probability as $\sum_{1}^{R_{R M}+Q_{R M}} n P_{n}$ where $P_{n}$ is the steady state probability that the subsystem is in state $n$. Therefore, the emissions formulation due to transportation and storage at RM stock, $E_{R M}$, is:

$$
E_{R M}=\frac{\varepsilon_{O} \mu_{d}(1)}{Q_{R M}}+\varepsilon_{H} \sum_{1}^{R_{R M}+Q_{R M}} n P_{n}
$$


Figure 5 State transition diagram for subsystem S(1) (see online version for colours)
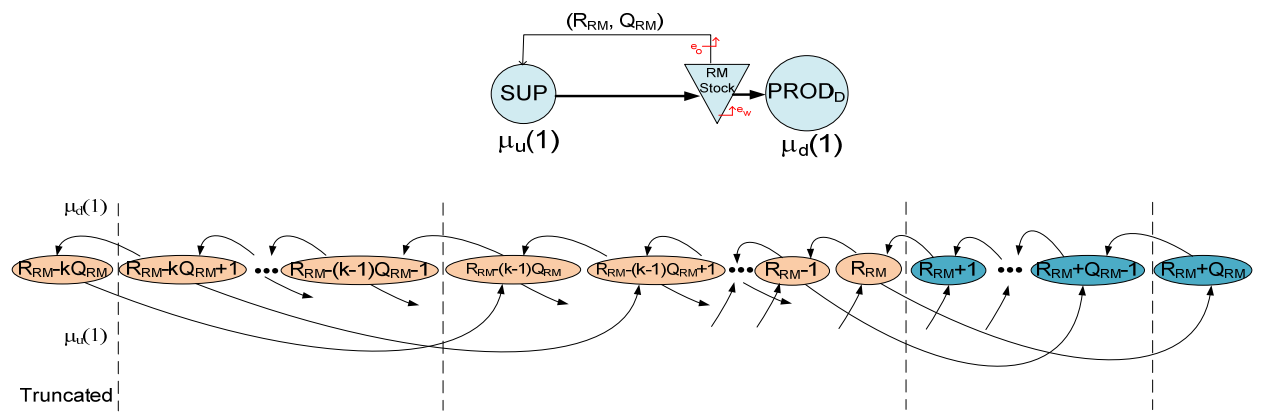

Total cost at RM stock, $T C_{R M}$ is given by:

$$
\begin{aligned}
T C_{R M}= & (\text { cost of ordering }+ \text { cost of holding }+ \text { cost of stockout }) \\
& + \text { cost of emission }
\end{aligned}
$$

Which can be formulated as:

$$
T C_{R M}=\frac{\mu_{d}(1) K_{R M}}{Q_{R M}}+C_{H} \sum_{1}^{R_{R M}+Q_{R M}} n P_{n}+\left|\frac{\mu_{d}(1) C_{S}}{Q_{R M}} \sum_{-\infty}^{0} n P_{n}\right|+C_{\varepsilon} E_{R M}
$$

Substituting $E_{R M}$ from (2) yields:

$$
\begin{aligned}
T C_{R M}= & \frac{\mu_{d}(1) K_{R M}}{Q_{R M}}+C_{H} \sum_{1}^{R_{R M}+Q_{R M}} n P_{n}+\left|\frac{\mu_{d}(1) C_{S}}{Q_{R M}} \sum_{-\infty}^{0} n P_{n}\right| \\
& +C_{\varepsilon}\left[\frac{\varepsilon_{O} \mu_{d}(1)}{Q_{R M}}+\varepsilon_{H} \sum_{1}^{R_{R M}+Q_{R M}} n P_{n}\right]
\end{aligned}
$$

Further manipulation gives (4b), which is the total cost incurred at the upstream side.

$$
T C_{R M}=\frac{\mu_{d}(1)}{Q_{R M}}\left(K_{R M}+\left|C_{S} \sum_{-\infty}^{0} n P_{n}\right|+C_{\varepsilon} \varepsilon_{O}\right)+\left(C_{H}+C_{\varepsilon} \varepsilon_{H}\right) \sum_{1}^{R_{R M}+Q_{R M}} n P n
$$

\subsection{Production process at factory}

Here we model the costs at the production stage. Costs are incurred due to production, inventory holding, stockout, and emissions. Figure 6 shows the transition diagram for production activities in the factory subsystem $\mathrm{S}(2)$. Production emissions of $\varepsilon_{P} \mathrm{~kg}$ carbon/product unit will occur at a time when there is a transition from state $R-1$ to state $R$. When the system is in a certain (positive) state, there is a storage emission $\varepsilon_{H} \mathrm{~kg}$ carbon/unit of product/time unit. Whereas, on transition from state $R$ to state $N, R-Q_{R}$, for example, emissions will arise due to delivery to retailers of $\varepsilon_{O} \mathrm{~kg}$ carbon/shipment. 
Figure 6 State transition diagram for subsystem S(2), (a) complete transition diagram

(b) transition diagram where stock reductions occur only in batches

(c) transition diagrams where stock addition and stock reductions occur in batches (see online version for colours)

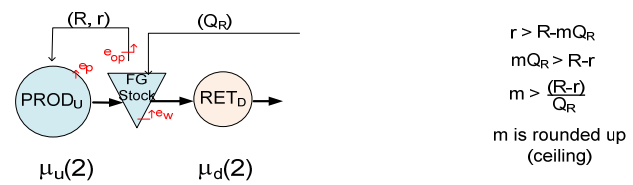

(a) $\mu_{\mathrm{u}}(2)$

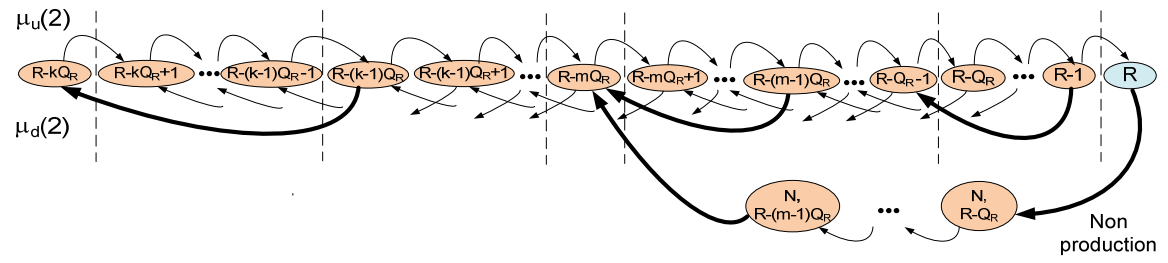

(b) $\mu_{\mathrm{u}}(2)$

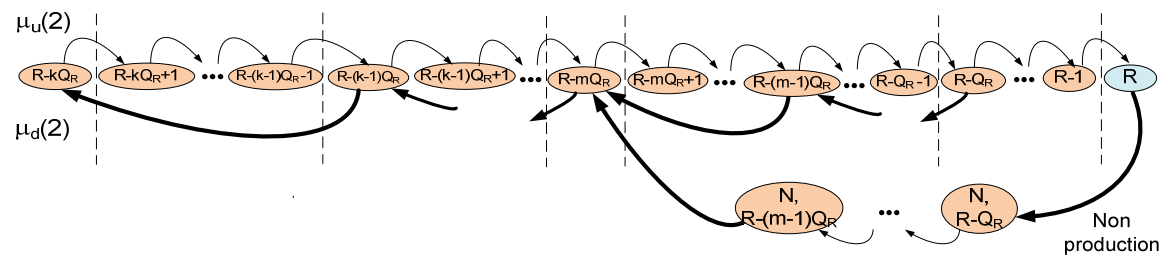

(c) $\mu_{\mathrm{u}}(2)$
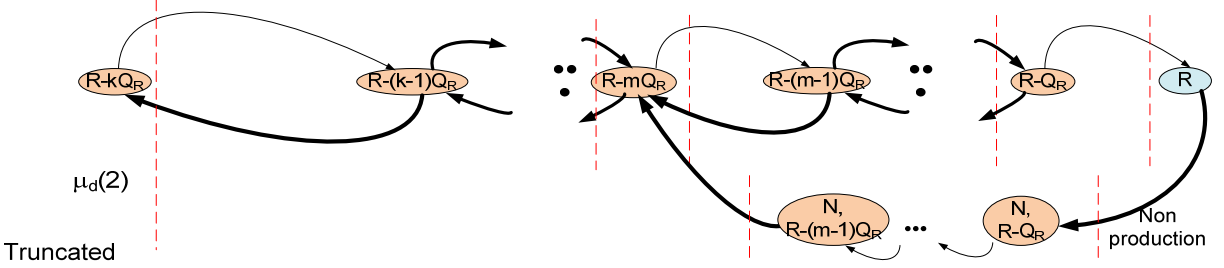

Total emissions in FG stock, $E_{F G}$, can be expressed as follows:

$E_{F G}=$ emissions due to production + emissions due to storage

Further manipulation gives:

$$
E_{F G}=\varepsilon_{P} \mu_{u}(2)+\varepsilon_{H}\left(\sum_{1}^{R} n P_{n}+\sum_{N, R-Q_{R}}^{n, R-(m-1) Q_{R}} n P_{n}\right)
$$

Total cost at FG stock, $T C_{F G}$, is given by:

$$
\begin{aligned}
T C_{F G}= & \text { cost of production }+ \text { cost of holding }+ \text { cost of stockout } \\
& + \text { cost of emission }
\end{aligned}
$$

With reference to Taha (2007), it can then be formulated that: 


$$
T C_{F G}=C_{P} \mu_{u}(2)+C_{H}\left(\sum_{1}^{R} n P_{n}+\sum_{N, R-Q_{R}}^{N, R-(m-1) Q_{R}} n P_{n}\right)+\left|\frac{C_{S} \mu_{d}(2)}{1} \sum_{-\infty}^{0} n P_{n}\right|+C_{\varepsilon} E_{F G}
$$

Substituting $E_{F G}$ from (6) yields:

$$
\begin{aligned}
T C_{F G}= & C_{P} \mu_{u}(2)+C_{H}\left(\sum_{1}^{R} n P_{n}+\sum_{N, R-Q_{R}}^{N, R-(m-1) Q_{R}} n P_{n}\right)+\left|\frac{C_{S} \mu_{d}(2)}{1} \sum_{1}^{0} n P_{n}\right| \\
& +C_{\varepsilon}\left[\varepsilon_{P} \mu_{u}(2)+\varepsilon_{H}\left(\sum_{1}^{R} n P_{n}+\sum_{N, R-Q_{R}}^{N, R-(m-1) Q_{R}} n P_{n}\right)\right]
\end{aligned}
$$

Further manipulation gives:

$$
\begin{aligned}
T C_{F G}= & \left(C_{P}+C_{\varepsilon} \varepsilon_{P}\right) \mu_{u}(2)+\left(C_{H}+C_{\varepsilon} \varepsilon_{H}\right)\left(\sum_{1}^{R} n P_{n}+\sum_{N, R-Q_{R}}^{N, R-(m-1) Q_{R}} n P_{n}\right) \\
& +\left|\frac{C_{S} \mu_{d}(2)}{1} \sum_{-\infty}^{0} n P_{n}\right|
\end{aligned}
$$

where $\frac{\mu_{d}(2)}{1}$ is the number of inventory cycles per unit of time. Number 1 (the denominator) is the production lot-size of this case.

\subsection{Shipment of products to the retailer's warehouse}

The state transition diagram for subsystem $S(3)$ is shown in Figure 7. An ordering activity from the retailer to the factory causes the state to transition, for example from state $R_{R}$ to state $R_{R}+Q_{R}$. Then, there will be emissions of $\varepsilon_{O} \mathrm{~kg}$ carbon/order. When the system is in a certain state, there is a storage emission of $\varepsilon_{H} \mathrm{~kg}$ carbon/unit of product/time. Emissions due to transition from state $R_{R}+Q_{R}$ to state $R_{R}+Q_{R}-1$ and so on, which represents the retail purchase process, are not taken into account.

Figure 7 State transition diagram for subsystem S(3) (see online version for colours)

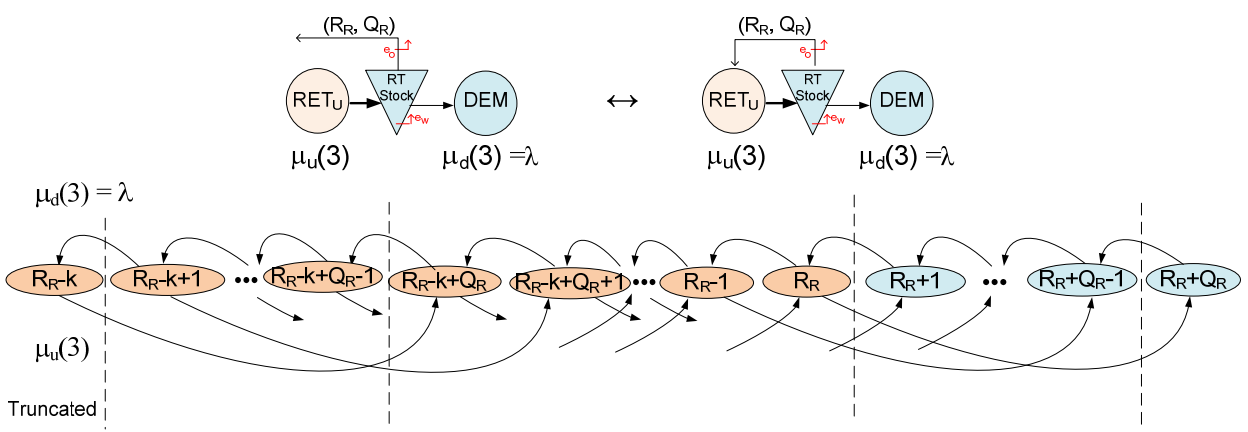


Total emission at RT stock, $E_{R T}$, is, therefore:

$$
\begin{aligned}
& E_{R T}=\text { emissions due to transportation }+ \text { emissions due to storage } \\
& E_{R T}=\frac{\lambda \varepsilon_{O}}{Q_{R}}+\varepsilon_{H} \sum_{1}^{R_{R}+Q_{R}} n P_{n}
\end{aligned}
$$

Where again, here we assume that one delivery is for one order and, hence, the frequency of order is equal to the frequency of delivery.

The total cost at RT stock, $T C_{R T}$ :

$$
\begin{aligned}
T C_{R T}= & \text { cost of ordering }+ \text { cost of holding }+ \text { cost of stockout } \\
& + \text { cost of emission } \\
T C_{R T}= & \frac{\lambda K_{R T}}{Q_{R}}+C_{H} \sum_{1}^{R_{R}+Q_{R}} n P_{n}+\left|\frac{\lambda C_{S}}{Q_{R}} \sum_{-\infty}^{0} n P_{n}\right|+C_{\varepsilon}\left(\frac{\lambda \varepsilon_{O}}{Q_{R}}+\varepsilon_{H} \sum_{1}^{R_{R}+Q_{R}} n P_{n}\right)
\end{aligned}
$$

which then gives:

$$
T C_{R T}=\left(K_{R T}+C_{\varepsilon} \varepsilon_{O}\right) \frac{\lambda}{Q_{R}}+\left(C_{H}+C_{H} \varepsilon_{H}\right) \sum_{1}^{R_{R}+Q_{R}} n P_{n}+\left|\frac{\lambda C_{S}}{Q_{R}} \sum_{-\infty}^{0} n P_{n}\right|
$$

\section{Numerical examples}

We consider a hypothetical case as follows. A supply chain system consists of one supplier, one factory, and one retailer, as shown in Figure 1. Transportation of goods from supplier to factory takes 50 units of time. The same amount of time is needed to transport finished goods from the factory to the retailer. Production can take place at a rate of 0.125 units/unit time, without the need for setup. Demand to the retailer is 0.02 units/unit time.

Lot-sizes and inventory buffering parameters are as follows: $R_{R M}=10 ; Q_{R M}=15$; $r=10 ; R=30 ; R_{R}=5 ; Q_{R}=10$. In addition, the cost parameters are as follows: ordering to supplier, $K_{R M}=2$ million IDR/order; storage at RM stock, $C_{h, R M}=1,000 \mathrm{IDR} /$ unit/unit time; shortage at RM stock, $C_{s, R M}=5,000 \mathrm{IDR} /$ unit; production, $C_{p}=50,000 \mathrm{IDR} /$ unit; storage in FG stock, $C_{h, F G}=1,000 \mathrm{IDR} /$ unit/unit time; shortage in FG stock, $C_{s, F G}=$ 5,000 IDR/unit; order to factory, $K_{R T}=2$ million IDR/order; storage in RT stock, $C_{h, R T}=$ 1,000 IDR/unit/unit of time; shortage in RT stock, $C_{s, R T}=5,000 \mathrm{IDR} /$ unit.

We also assume that carbon tax, $C_{\varepsilon}=270 \mathrm{IDR} / \mathrm{kg}$ carbon. The emission factor data are set as follows. Transport emissions from supplier to factory, $\varepsilon_{O, R M}=500 \mathrm{~kg}$ carbon; storage emission at RM stock, $\varepsilon_{H, R M}=0.05 \mathrm{~kg}$ carbon/product unit/time unit; production emission $\varepsilon_{P}=0.5 \mathrm{~kg}$ carbon/product unit; storage emission at FG stock, $\varepsilon_{H, F G}=0.05 \mathrm{~kg}$ carbon/product unit/time; shipping emission from factory to retailer, $\varepsilon_{O, R T}=300 \mathrm{~kg}$ carbon; and storage emissions at RT stock, $\varepsilon_{H, R T}=0.05 \mathrm{~kg}$ carbon/product unit/time unit.

The following results are obtained: average inventory at RM stock $=16.99$ units, average inventory at FG stock $=23.34$ units, and average inventory at RT stock $=9.51$ units. The cost of emission at RM stock $=4,221.00 \mathrm{IDR} /$ time unit, emission cost in FG stock $($ production $)=3,015.44 \mathrm{IDR} /$ time unit, and emission cost in $\mathrm{RT}$ stock $=3,022.44$ 
IDR/time unit. In addition, the total cost that includes inventory-related costs and emission cost in $\mathrm{RM}$ stock $=24,821.00 \mathrm{IDR} /$ time unit, in FG stock (production) $=$ 30,352.06 IDR/time unit, and in RT stock $=17,410.06 \mathrm{IDR} /$ time unit. This gives total costs of $72,583.12 \mathrm{IDR} /$ time unit.

\section{Discussion}

In this paper, emissions, as well as costs in this case, can only be accommodated in the form of a response variable. Hence, the cost curve pattern, which includes inventory-related costs, emissions costs, and total costs, is illustrated in the form of plot resulted from experiments using models.

Figure 8 Result of calculation of total cost and emission cost, on various variation of decision value of (a) $R_{R M}$ (b) $Q_{R M}$ (c) $r$ (d) $R$ (e) $R_{R}$ (f) $Q_{R M}$ (see online version for colours)

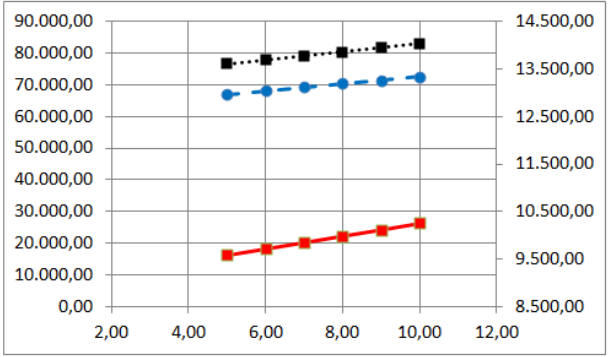

(a)

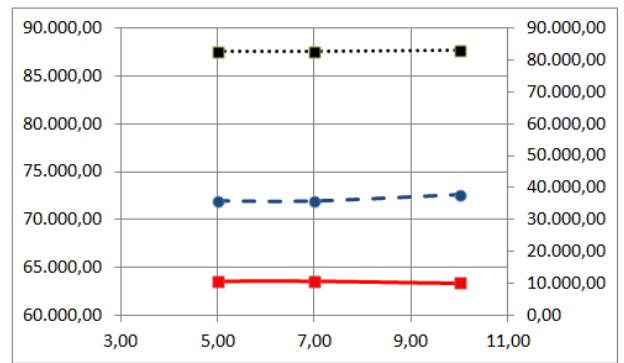

(c)

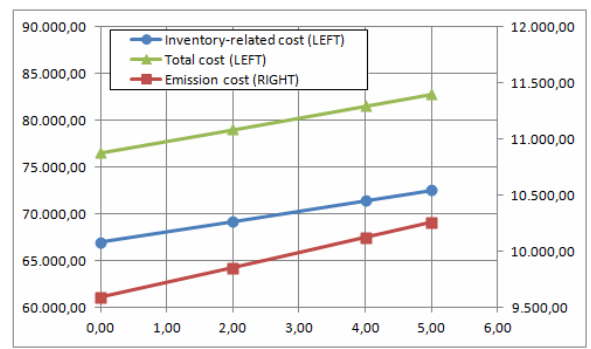

(e)

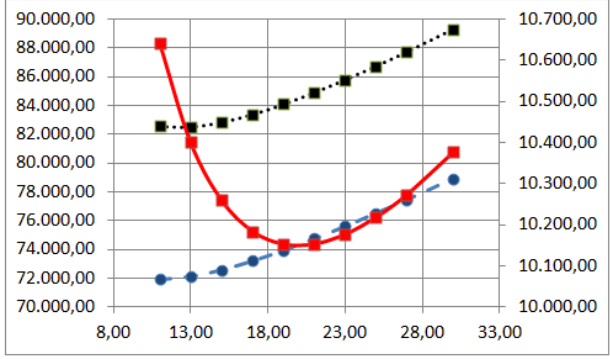

(b)

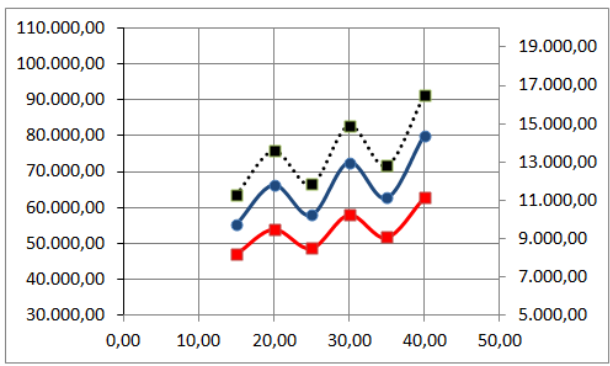

(d)

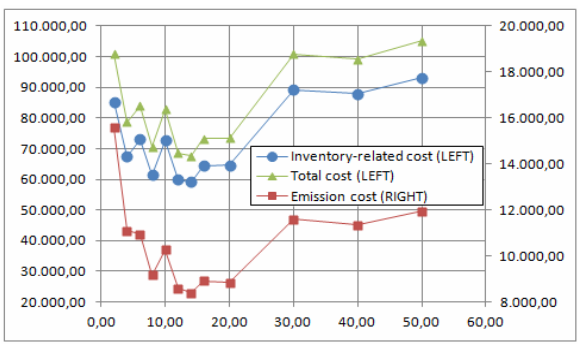

(f)

Notes: -८- Inventory-related cost (LEFT)

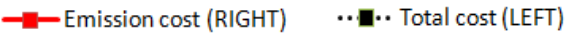


For a particular configuration and decision, the system will operate by generating emission of a certain amount of $\mathrm{kg}$ carbon per unit of time. The computation results for some values of decision variables in the hypothetical case above are summarised in Figure 8 .

Varying the various values of decision variables $R_{R M}, Q_{R M}, r, R, R_{R}$ and $Q_{R}$ yield some interesting observations. First, the variation of reorder point values $R_{R M}$ and $R_{R}$ gives the effect of increasing total costs. This is logical, because the increase in reorder point will lead to an increase in the level of stocks in the system and the frequency of ordering. As we assume that each order is associated with a single shipment, altering the frequency of orders is not only affecting the inventory related cost, but also the level of emissions. Interestingly, the increase in reproduction point $r$ has no impact on the total cost. This is due to the assumption that there is no set-up cost every time the production is restarted and the production cost only consists of variable cost.

Second, the increase of order quantity value, $Q_{R M}$ and $Q_{R}$ gives a similar effect, i.e., there is a certain order quantity value which will minimise the total cost. However, there is a different pattern of response between total cost and emission cost, when $Q_{R M}$ increases [Figure $8(\mathrm{~b})$ ]. The emission cost appears to reach the minimum value at a much higher $Q_{R M}$, indicating that considering emission costs is important as it cannot be represented by the total inventory-related costs. In this example, the optimum $Q_{R M}=12$ (based on total cost). It would have been 11 if the criterion is to minimise the inventory related costs only, but the value would have been 20 if the criterion is to minimise emission costs only.

A largely different thing happened to $Q_{R}$. Besides its fluctuating profile, the patterns of response of inventory-related and emission cost are almost similar. If the total cost is used as a reference, then $Q_{R}=12$. If inventory-related cost is used, then $Q_{R}=12$ too, and, if only emission cost is considered, then again $Q_{R}=12$ [Figure 8(f)]. This observation is obviously dependent on the relative values of the cost parameters. However, it is obvious to note here that considering emission as a response variable in addition to the inventory-related costs would lead to a different decision.

Meanwhile, the increase of $R$ value gives a quite unique impact, that is, there is a general trend of rising in the total inventory-related cost as well as the emission costs, but a closer look shows that those two response variables oscillate with the increase of $R$. As $R$ increases, the value of $(R-r) / Q_{R}$ gets larger, causing the ordering frequency of retailers within that range to expand. When the ordering frequency increases, the total cost will also increase. However, at a certain $R$ value, the stock level target could be reached, causing inventory reduction at FG stock to occur while there is no production and, hence, no production emissions for a certain period of time. When that happens, the total cost becomes lower.

\section{Conclusions}

Carbon emission issues should be accommodated as a criterion for decision making in industry or supply chain systems. Certain supply chain activities lead directly to carbon emissions. Ordering activities are almost always related to the transport of goods from one location to another using various transportation modes. Since most of the current transportation still uses fossil fuels, carbon emissions always occur. In addition, production and storage activities also create emissions when fossil fuel is used as their 
primary energy source and, thus, considering emissions in those activities would be important.

In this paper, we developed a supply chain model that considers both inventory-related costs and emissions. We used the queuing-based performance model where emissions are captured in the delivery of materials from supplier to factory, at the production activity, and at the storage of goods, both for raw materials as well as for finished goods. We contribute to the supply chain modelling works where only very few queuing-based performance models have taken carbon emissions into account. In solving the model, in this paper, we have used the decomposition approach following the works of Dallery and Frein (1993) and Karaman and Altiok (2009). Experiments were conducted to test the applicability of the model. For model validation, we have used a discrete event simulation. The computation results show that the two results (the decomposition approach and the simulation) are very close, indicating the accuracy of the approach that we used. The numerical examples show that the change in parameter values is not always responded the same way by the total inventory-related costs and the emission costs, indicating the significance of the model that takes into account both of these response variables.

Future work can be extended to supply chain systems consisting of multiple suppliers and some retailers. First, a more complex supply chain structure may be considered. For example, distribution centres can be added in order to reflect a more realistic situation. Second, it is interesting to consider multi-modal transportation decisions. Obviously, there will be trade-offs between speed, cost, flexibility and emissions. For example, rail normally creates fewer emissions compared to truck, but, at the same time, is less flexible and more costly if used for transporting over a relatively short period. Models considering multiple transportation modes may be worth developing in order to guide the decision makers under a complex decision-making situation.

\section{References}

Altiok, T. (1997) Performance Analysis of Manufacturing Systems, Springer Science+Business Media, New York.

Arslan, M.C. and Turkay, M. (2013) 'EOQ revisited with sustainability considerations', Foundations of Computing and Decision Sciences, Vol. 38, No. 4, pp.223-249.

Benjaafar, S., Li, Y. and Daskin, M. (2012) 'Carbon footprint and the management of supply chains: insights from simple models', IEEE Transactions on Automation Science and Engineering, Vol. 10, No. 1, pp.1-18.

Brandenburg, M. (2015) 'Low carbon supply chain configuration for a new product - a goal programming approach', International Journal of Production Research, Vol. 53, No. 21, pp.6588-6610, DOI: 10.1080/00207543.2015.1005761.

Carbonfootprint.com (n.d.) Climate Change [online] http://www.carbonfootprint.com/ warming.html (accessed 13 December 2016).

Chen, X., Benjaafar, S. and Elomri, A. (2013) 'The carbon-constrained EOQ', Operations Research Letters, Vol. 41, No. 2, pp.172-179.

Dallery, Y. and Frein, Y. (1993) 'On decomposition methods for tandem queueing networks with blocking', Operations Research, Vol. 41, No. 2, pp.386-399.

Dallery, Y. and Gershwin, S.B. (1992) 'Manufacturing flow line systems: a review of models and analytical results', Queueing Systems, Vol. 12, Nos. 1-2, pp.3-94. 
Fahimnia, B., Sarkis, J. and Eshragh, A. (2015a) 'A tradeoff model for green supply chain planning: a leanness-versus-greenness analysis', Omega (United Kingdom), Vol. 54, pp.173-190, doi:10.1016/j.omega.2015.01.014.

Fahimnia, B., Sarkis, J., Choudhary, A. and Eshragh, A. (2015b) 'Tactical supply chain planning under a carbon tax policy scheme: a case study', International Journal of Production Economics, Vol. 164, pp.206-215, doi:10.1016/j.ijpe.2014.12.015.

Gershwin, S.B. (1987) 'An efficient decomposition method for the approximate evaluation of tandem queues with finite storage space and blocking', Operations Research, Vol. 35, No. 2, pp.291-305.

Hua, G., Cheng, T.C.E. and Wang, S. (2011) 'Managing carbon footprints in inventory management', International Journal of Production Economics, Vol. 132, No. 2, pp.178-185.

Karaman, A. and Altiok, T. (2009) 'Approximate analysis and optimization of batch ordering policies in capacitated supply chains', European Journal of Operational Research, Vol. 193, No. 1, pp.222-237 [online] doi:10.1016/j.ejor.2007.10.018.

Murdapa, P.S., Pujawan, I.N., Karningsih, P.D. and Nasution, A.H. (2018) 'Single stage queueing/manufacturing system model that involves emission variable', IOP Conference Series: Materials Science and Engineering, Vol. 337.

Pan, S., Ballot, E. and Fontane, F. (2013) 'The reduction of greenhouse gas emissions from freight transport by pooling supply chains', International Journal of Production Economics, Vol. 143, No. 1, pp.86-94 [online] http://dx.doi.org/10.1016/j.ijpe.2010.10.023.

Ratnatunga, J., Wahyuni, D. and Jones, S. (2012) 'The carbon economy and emissions reporting', Contemporary Issues in Sustainability Accounting, Assurance and Reporting, pp.153-182, Emerald, Bingley, England.

Saetta, S., Paolini, L., Tiacci, L. and Altiok, T. (2012) 'A decomposition approach for the performance analysis of a serial multi-echelon supply chain', International Journal of Production Research, Vol. 50, No. 9, pp.2380-2395.

Shaw, K., Shankar, R., Yadav, S.S. and Thakur, L.S. (2013) 'Modeling a low-carbon garment supply chain', Production Planning \& Control, Vol. 24, Nos. 8-9, pp.851-865.

Taha, H.A. (2007) Operations Research: An Introduction, 8th ed., Pearson Prentice Hall, Upper Saddle River NJ.

Toptal, A. and Çetinkaya, B. (2015) 'How supply chain coordination affects the environment: a carbon footprint perspective', Annals of Operations Research, Vol. 250, No. 2, pp.487-519.

Toptal, A., Özlü, H. and Konur, D. (2014) 'Joint decisions on inventory replenishment and emission reduction investment under different emission regulations', International Journal of Production Research, Vol. 52, No. 1, pp.243-269.

US Environmental Protection Agency (2016) Climate Change Indicators in the United States, 2016, 4th ed., EPA 430-R-16-004 [online] http://www.epa.gov/climate-indicators (accessed 8 February 2017).

Wang, X.J. and Choi, S.H. (2015a) 'Stochastic lot sizing for shareholder wealth maximisation under carbon footprint management', Journal of Industrial and Intelligent Information, Vol. 3, No. 1, pp.1-8.

Wang, X.J. and Choi, S.H. (2015b) 'Impacts of carbon emission reduction mechanisms on uncertain make-to-order manufacturing, International Journal of Production Research, April, Vol. 54, No. 11, pp.1-18, doi:10.1080/00207543.2015.1106606.

Zakeri, A., Dehghanian, F., Fahimnia, B. and Sarkis, J. (2015) 'Carbon pricing versus emissions trading: a supply chain planning perspective', International Journal of Production Economics, Vol. 164, pp.197-205. 